\title{
BRAVEHeart: A randomised trial comparing the accuracy of Breathe Well and RPM for deep inspiration breath hold breast cancer radiotherapy
}

Hilary Byrne ( $\sim$ hilary.byrne@sydney.edu.au )

The University of Sydney School of Health Sciences https://orcid.org/0000-0001-7023-7976

Elisabeth Steiner

Landesklinikum Wiener Neustadt

Jeremy Booth

Royal North Shore Hospital Northern Sydney Cancer Centre

Gillian Lamoury

Royal North Shore Hospital Northern Sydney Cancer Centre

Marita Morgia

Royal North Shore Hospital Northern Sydney Cancer Centre

Kylie Richardson

Royal North Shore Hospital Northern Sydney Cancer Centre

Leigh Ambrose

Royal North Shore Hospital Northern Sydney Cancer Centre

Kuldeep Makhija

The University of Sydney School of Health Sciences

Cameron Stanton

Royal North Shore Hospital Northern Sydney Cancer Centre

Ben Zwan

Royal North Shore Hospital Northern Sydney Cancer Centre John Atyeo

Royal North Shore Hospital Northern Sydney Cancer Centre

Shona Silvester

The University of Sydney School of Health Sciences

Natalie Plant

The University of Sydney School of Health Sciences

Paul Keall

The University of Sydney School of Health Sciences 
Keywords: Breast cancer, radiotherapy, deep inspiration breath hold, motion monitoring, visual feedback, surface monitoring

Posted Date: February 11th, 2022

DOI: https://doi.org/10.21203/rs.3.rs-779403/v1

License: (1) This work is licensed under a Creative Commons Attribution 4.0 International License. Read Full License 


\section{Abstract}

Background: Deep inspiration breath hold (DIBH) reduces radiotherapy cardiac dose for left-sided breast cancer patients. The BRAVEHeart (Breast Radiotherapy Audio Visual Enhancement for sparing the Heart) trial will assess the accuracy and usability of a novel device, Breathe Well, for DIBH guidance for leftsided breast cancer patients. Breathe Well will be compared to an adapted widely available monitoring system, the Real-time Position Monitoring system (RPM).

Methods: BRAVEHeart is a single institution prospective randomised trial of two DIBH devices. BRAVEHeart will assess the DIBH accuracy for Breathe Well and RPM during left-sided breast cancer radiotherapy. After informed consent has been obtained, 40 patients will be randomised into two equal groups, the experimental arm (Breathe Well) and the control arm (RPM with in-house modification of an added patient screen). The primary hypothesis of BRAVEHeart is that the accuracy of Breathe Well in maintaining the position of the chest during DIBH is superior to the RPM system. Accuracy will be measured by comparing chest wall motion extracted from images acquired of the treatment field during breast radiotherapy for patients treated using the Breathe Well system and those using the RPM system.

Discussion: The Breathe Well device uses a depth camera to monitor the chest surface while the RPM system monitors a block on the patient abdomen. The hypothesis of this trial is that the chest surface is a better surrogate for the internal chest wall motion used as a measure of treatment accuracy. The Breathe Well device aims to deliver an easy to use implementation of surface monitoring. The findings from the study will help inform the technology choice for other centres performing DIBH.

Trial registration: This trial was registered on ClinicalTrials.gov on 26 August 2016. The identifier is: NCT02881203. (https://clinicaltrials.gov/ct2/show/NCT02881203).

\section{Administrative Information}

\section{Note}

the numbers in curly brackets in this protocol refer to SPIRIT checklist item numbers. The order of the items has been modified to group similar items (see http://www.equator-network.org/reportingguidelines/spirit-2013-statement-defining-standard-protocol-items-for-clinical-trials/). 


\section{Title $\{1\} \quad$ BRAVEHeart: A randomised trial comparing the accuracy of Breathe Well and RPM for deep inspiration breath hold breast cancer radiotherapy}

Trial registration $\{2 a$ and $2 \mathrm{~b}\}$.
This trial was registered on ClinicalTrials.gov on 26 August 2016. The identifier is: NCT02881203.

https://clinicaltrials.gov/ct2/show/NCT02881203
Protocol

Funding $\{4\}$ version $\{3\}$

v3.0, 13th August 2020

The BRAVEHeart trial is funded by the National Health and Medical Research Council (NHMRC) Development Grant (APP1073772) and the National Breast Cancer Foundation of Australia Pilot Study Grant (PS-17-055).

Author details $\{5 a\}$
Hilary Byrne ${ }^{a}$, Elisabeth Steiner ${ }^{b}$, Jeremy Booth ${ }^{c, d}$, Gillian Lamouryc $^{c}$, Marita Morgiac, Kylie Richardsonc, Leigh Ambrosec, Kuldeep Makhija a , Cameron Stantonc, Benjamin Zwan', Felix Bockelmann" ${ }^{c}$, Regina Bromley ${ }^{c}$, John Atyeo ${ }^{c}$, Shona Silvester ${ }^{\mathrm{a}}$, Natalie Plant ${ }^{\mathrm{a}}$, Paul Keall ${ }^{\mathrm{a}}$

a ACRF Image X Institute, School of Health Sciences, The University of Sydney, Sydney, Australia

b LK Wiener Neustadt, Austria

${ }^{c}$ Northern Sydney Cancer Centre, Royal North Shore Hospital, Sydney, Australia

d School of Physics, The University of Sydney, Australia

Name and contact information for the trial sponsor $\{5 b\}$

Role of sponsor $\{5 \mathrm{c}\}$
The University of Sydney, Sydney, NSW, 2006, Australia

\begin{abstract}
The study sponsor and funders will not influence study design; collection, management, analysis, and interpretation of data; writing of the report; the decision to submit the report for publication.
\end{abstract}

\section{Introduction}

\section{Background and rationale $\{6 a\}$}

Radiotherapy is recommended for $87 \%$ of all diagnosed breast cancer patients for disease control [1], but it can lead to late cardiac side effects [2]. Deep inspiration breath hold (DIBH) increases the lung volume and moves the heart outside of the irradiation fields, reducing the heart dose [3-7] and thus reducing the cardiac toxicity during breast cancer radiotherapy $[2,8,9]$. Nissen et al. [7] estimated that DIBH reduces the mean heart dose from 5.2 to $2.7 \mathrm{~Gy}$ on average and Darby et al. [2] concluded that the rate of major coronary events increased linearly by $7.4 \%$ per Gray of mean heart dose with no threshold. The Darby data outlines that it is important to keep the dose to the heart from radiotherapy, especially for young 
patients, as low as possible and based on their analysis DIBH reduces the increased rate of major coronary events due to the radiation treatment by $20 \%$ [2].

Standardly the patients are guided to the breath hold level with audio prompts from the operating radiation therapists. However, Cervino et al. [10] demonstrated that providing the patients with visual feedback by adding a monitor or goggles in addition to audio guidance has demonstrated improvement in the reproducibility of DIBH by $95 \%$ and stability by $80 \%$ (and for variations $>2 \mathrm{~mm}$ by $35 \%$ and $15 \%$, respectively). Similar findings have been reported by Damkjær et al. [11].

Several systems are currently commercially available to assist the implementation of DIBH in the clinic. These systems include: the real-time position management (RPM) system and real-time gating for scanners (RGSC) system (Varian Medical Systems, Palo Alto, CA), the active breathing coordinator (ABC) (Elekta, Stockholm, Sweden), AlignRT (Vision RT Inc., London, UK), Catalyst HD (C-RAD, Uppsala, Sweden), Exac-Trac (Brainlab, Feldkirchen, Germany), and Identify (Varian Medical Systems, Palo Alto, CA).

RPM and RGSC utilise an infrared camera tracking a marker block, standardly placed on or near the xyphoid process on the abdomen (Figure 2). ABC uses a spirometer with a valve that is closed for the breath hold. AlignRT, Catalyst HD, Exac-Trac and Identify use 3D surface imaging, requiring in-room installation and/or do not standardly include a visual feedback feature.

Breathe Well is a new audiovisual (AV) feedback device developed at the University of Sydney that may increase the efficacy and improve the workflow of implementing DIBH for breast cancer patients (Figure 1). Breathe Well is clamped on the superior end of the computed tomography (CT) couch or treatment couch and uses depth and optical sensors to directly monitor a user-defined area on a patient's chest. The positional accuracy and precision of the depth sensor has been shown to be within $\pm 1.0 \mathrm{~mm}$ and the temporal stability of the sensor is sub-mm [12]. The horizontal and vertical axes of the device can be adjusted to fit the patient and immobilisation device dimensions and the device arm can be rotated to enable an unobstructed patient setup and an optimal view of the included screen when the patients are treated with their head tilted. Within the Breathe Well software application a patient-specific reference breath hold with respect to the normal breathing baseline can be measured and stored. The included screen delivers real-time feedback to the patient regarding their chest position. The breath hold target area is highlighted and depending on the workflow status the screen shows an orange wait screen or instructs the patient to hold their breath or relax. An optional breath hold countdown option helps the patient to see how long they still have to hold their breath. At their workstation, the radiation therapists also see the real-time visual feedback indicating when the breath hold depth is acceptable, allowing them to control treatment delivery.

In this study, Breathe Well will be tested as a new candidate for DIBH guidance and monitoring.

\section{Objectives $\{7\}$}


The primary objective of the study is to test the accuracy and usability of Breathe Well to assist DIBH during breast cancer radiotherapy. Breathe Well will be tested against the commercially available realtime position management (RPM) / respiratory gating for scanners (RGSC) system (Varian Medical Systems, Palo Alto, CA), that has been extended in-house to provide a screen for visual feedback.

The primary hypothesis is that the accuracy of Breathe Well in maintaining the position of the chest during DIBH is superior to the RPM/RGSC system. Because Breathe Well monitors the chest directly, while the RPM/RGSC system uses a marker block positioned on the upper abdomen, Breathe Well has the advantage of utilising a more immediate surrogate of the chest wall position. In addition, we hypothesise that Breathe Well is more user-friendly as it was specifically designed for guiding breast cancer patients through DIBH treatments.

Secondary objectives:

1. To measure the setup times for both the Breathe Well and the modified RPM/RGSC system for all fractions. We hypothesise that Breathe Well can be set up faster than the modified RPM/RGSC system.

2. To investigate patient comfort and user friendliness of Breathe Well via a patient survey (Supplementary Materials). We hypothesise that the patient comfort and usability of Breathe Well is superior to the Varian RPM system.

3. To investigate staff perception of Breathe Well via a technology assessment survey (Supplementary Materials). We hypothesise that the staff perception of Breathe Well is superior to the Varian RPM/RGSC system.

4. To develop - offline - the use of the electronic portal imaging device (EPID) for real-time MLC tracking during monitoring the chest wall position in breast radiotherapy.

5. To investigate, using dose reconstruction, the estimated dose delivered during radiotherapy and compare this with the planned dose.

\section{Trial design \{8\}}

The trial design is a randomised, prospective superiority trial with an experimental (breathe well) and a control (RPM/RGSC) arm allocation ratio 1:1. Patients receiving radiotherapy for left-sided breast cancer will be randomised into two equal groups. The experimental arm will manage breath holds with audiovisual biofeedback from the Breathe Well device. Patients in the control arm will manage breath holds using an in-house developed audiovisual feedback for the RPM/RGSC system. The trial schema is summarised in Figure 3.

\section{Methods: Participants, Interventions And Outcomes}

\section{Study setting $\{9\}$}


Study participants will be recruited and treated at the Royal North Shore Hospital, St Leonards, NSW, 2065, Australia.

\section{Eligibility criteria $\{10\}$}

Inclusion - Patients

1. Left-sided breast cancer patients (invasive and in situ)

2. To be treated in the supine position

3. Ability to perform $a \geq 20$ s breath hold

4. $>18$ years old

5. Female

6. An ECOG score from 0 to 2

7. Able to read and complete questionnaires in English

8. Able to give written informed consent and willingness to participate and comply with the study

Exclusion - Patients

1. No regional lymph nodes involved or at risk

2. No pregnant / lactating women

\section{Who will take informed consent? $\{26 a\}$}

Potential participants will be identified by their consulting clinician who will explain the study, including risks and benefits and give the opportunity for informed discussion. They will receive a copy of the informed consent form to carefully read and take home for further analysis if necessary. Trained clinical trials staff at Royal North Shore Hospital will obtain informed consent from patients prior to their CT simulation appointment.

\section{Additional consent provisions for collection and use of participant data and biological specimens $\{26 \mathrm{~b}\}$}

Not applicable.

\section{Interventions}

\section{Explanation for the choice of comparators $\{6 \mathrm{~b}\}$}

The choice was made to compare the novel Breathe Well device to RPM/RGSC as an existing widely adopted commercial system in clinical use for breath hold management.

\section{Intervention description $\{11$ a $\}$}

Training and simulation: The patients will be trained to perform DIBH with the assigned system and assessed for ability to perform a 20 second breath hold (at $80-90 \%$ of the maximal lung capacity). Based 
on this screening the patient will be deemed suitable for DIBH. For patients suitable for DIBH the breath hold AV guidance while acquiring the planning CT scan will be provided by the assigned system. The unallocated system will be set up to passively record motion, not interfering with simulation in any way. The reference breath hold depth will be recorded for both systems.

Treatment delivery: For treatment, similarly to simulation, both systems will be set up to monitor breathing motion. The patient will be guided with AV biofeedback from the assigned system depending which arm of the trial they have been randomised to. The unallocated system will passively acquire data but not interfere with treatment in any way. Prior tests showed that no interferences and difficulties are anticipated when operating the 'Breathe Well' system and the RPM/RGSC system at the same time. If any treatment fraction cannot be delivered due to a failure of the Breathe Well device, this will be recorded. The treatment will continue at the physician's discretion either (1) using the RPM system, (2) the fraction will be rescheduled or (3) delivered using free breathing.

\section{Criteria for discontinuing or modifying allocated interventions $\{11 \mathrm{~b}\}$}

If the patient is not suitable for DIBH, the standard clinical workflow for free breathing (FB) is followed. The patient will complete the patient experience questionnaire and progress to treatment with free breathing using no breath hold management system and with no further study interventions.

\section{Strategies to improve adherence to interventions $\{11 c\}$}

Not applicable. Assessment of ease of use of treatment with the Breathe Well device is the aim of this trial, so non-adherence to the intervention should be recorded. Inability to carry out DIBH will result in freebreathing treatment as per current standard of care.

\section{Relevant concomitant care permitted or prohibited during the trial $\{11 \mathrm{~d}\}$}

No restrictions on concomitant care being offered as per standard hospital practice.

\section{Provisions for post-trial care $\{30\}$}

Participants will receive post-treatment care as per standard hospital procedure. Compensation could be available through sponsor insurance.

\section{Outcomes $\{12\}$}

The primary outcome of this study is a comparison of the accuracy of the novel device Breathe Well compared to existing clinical standard RPM/RGSC, with data to be analysed after completion of treatment.

\section{Participant timeline $\{13\}$}


Table 1 shows the schedule of enrolment, interventions, and assessments. Initial toxicity baseline will be taken at enrolment, and again at post-treatment follow up at 6 weeks and every 6 months for three visits.

Table 1: schedule of enrolment, interventions, and assessments

\begin{tabular}{|c|c|c|c|c|c|c|}
\hline \multirow[b]{3}{*}{ TIMEPOINT } & \multicolumn{6}{|c|}{ STUDY PERIOD } \\
\hline & \multirow{2}{*}{$\begin{array}{l}\text { Enrolment } \\
\text { Initial } \\
\text { consult }\end{array}$} & \multirow{2}{*}{$\begin{array}{l}\text { Allocation } \\
\text { CT } \\
\text { appoint. }\end{array}$} & \multicolumn{3}{|c|}{ Post-allocation } & \multirow{2}{*}{$\begin{array}{l}\text { Follow } \\
\text { up } \\
6 \mathrm{w} \\
\text { then } \\
\text { every } \\
6 \mathrm{~m}\end{array}$} \\
\hline & & & $\begin{array}{l}\text { Prior to } \\
\text { Treatment }\end{array}$ & $\begin{array}{l}\text { First } \\
\text { treatment }\end{array}$ & $\begin{array}{l}\text { Last } \\
\text { treatment }\end{array}$ & \\
\hline \multicolumn{7}{|l|}{ ENROLMENT: } \\
\hline Eligibility screen & $x$ & & & & & \\
\hline Informed consent & & $x$ & & & & \\
\hline Allocation & & $x$ & & & & \\
\hline \multicolumn{7}{|l|}{ INTERVENTION: } \\
\hline $\begin{array}{l}\text { DIBH training and } \\
\text { assessment }\end{array}$ & & $x$ & & & & \\
\hline CT scan & & $x$ & & & & \\
\hline Radiotherapy planning & & & $x$ & & & \\
\hline \multicolumn{7}{|l|}{$\begin{array}{l}\text { Radiotherapy treatment } \\
\text { with Breathe Well, } \\
\text { RPM/RGSC or free } \\
\text { breathing }\end{array}$} \\
\hline \multicolumn{7}{|l|}{ ASSESSMENTS: } \\
\hline $\begin{array}{l}\text { History \& physical exam } \\
\text { Toxicity will be } \\
\text { assessed at each } \\
\text { scheduled history and } \\
\text { physical exam visit }\end{array}$ & $x$ & & & & & $x$ \\
\hline $\begin{array}{l}\text { Ease of use } \\
\text { questionnaires }\end{array}$ & & $\mathrm{x}$ & & $\mathrm{x}$ & $\mathrm{x}$ & \\
\hline
\end{tabular}

\section{Sample size $\{14\}$}

The sample size was estimated by assuming that the systematic error in treatment accuracy, $\Sigma$, will be smaller for patients on the experimental (Breathe Well) arm than the control (RPM) arm. Previously published data for a different surface monitoring system (AlignRT: VisionRT, London, UK) is given by Kanphet et al. [18] who found a systematic error of $0.46 \mathrm{~mm}$ in the vertical direction. For comparison, 
Lutz et al. [19] found a systematic error of $1.33 \mathrm{~mm}$ with RPM. Assuming this study will give similar results, and using an F-test for equality of two variances, 36 patients will be needed for statistical significance with a power of 0.8 and alpha error probability of 0.05 . The sample size for this study was set at 40 to account for an estimated $10 \%$ drop out rate for patients who cannot maintain a breath hold and are treated with free breathing.

\section{Recruitment \{15\}}

Recruitment rate is expected to be relatively high due to the non-invasive nature of the intervention proposed, the fact that no extra appointments are required and no extra radiation dose will be delivered, and the number of eligible patients treated at the site.

\section{Assignment of interventions: allocation}

\section{Sequence generation $\{16 a\}$}

A total of 40 patients will be recruited. After an eligibility assessment and informed consent, participants will be assigned to the experimental arm or control arm with allocation ratio 1:1. The randomisation sequence and grouping will be generated by one researcher who will not be involved directly in patient screening, enrolment or assessment, and uploaded to Research Electronic Data Capture (REDCap). No stratification will be utilised. The randomisation tables were set up using permuted blocks with random varying block sizes of 4 and 6 .

\section{Concealment mechanism $\{16 \mathrm{~b}\}$}

The BRAVEHeart trial is randomised but not blinded. As the trial is to use one device versus another, the arm a patient is assigned to cannot be concealed to the patient or the treatment team.

\section{Implementation \{16c\}}

Randomisation is performed at the site electronically by the hospital clinical trials staff using REDCap. The allocation sequence is locked down within the REDCap database and is not accessible to anyone.

\section{Assignment of interventions: Blinding}

\section{Who will be blinded $\{17 a\}$}

No blinding will be used in this trial.

\section{Procedure for unblinding if needed $\{17 b\}$}

Not applicable. 


\section{Data collection and management}

\section{Plans for assessment and collection of outcomes $\{18 \mathrm{a}\}$}

Collection of data during treatment will be initiated by the radiation therapists delivering the treatment. Clinical trials staff will administer the ease of use questionnaires (see Additional Files 2 and 3 ) with the results entered in REDCap. Clinicians will administer baseline and toxicity questionnaires, with the results entered in REDCap.

\section{Plans to promote participant retention and complete follow-up $\{18 \mathrm{~b}\}$}

Participants are only required to attend treatment and follow-up appointments as normally required for their treatment and no drop out is expected. Clinicians and radiotherapy staff will be trained to answer any participant questions or concerns about the trial.

\section{Data management $\{19\}$}

Respiratory data ('Breathe Well' and RPM/RGSC), CT images, MV frames from during the irradiation, and demographic information will be collected from the subjects. At the randomisation stage of the study, patients will receive a trial ID. The data saved for the trial will be under this de-identified trial ID. A separate key of the subject study number and their medical record number will be securely stored by the chief investigator to allow re-identification if necessary. This master list for re-identification will remain at the Royal North Shore Hospital. Only the principal investigator will have the ability to re-identify subjects. Questionnaire and toxicity information will be entered in REDCap and collated information transferred to the same secure drive at the University of Sydney for storage. The data will be stored for 15 years as per clinical trial guidelines. Data sent from the Northern Sydney Cancer Centre, Royal North Shore Hospital in accordance with the study site's ethics and security allowances and protocols to the study site University of Sydney will be anonymised but re-identifiable. Patient data could be made reidentifiable to obtain additional clinical information for the data analysis stage of the project, but only by the principal investigator. The de-identified data will be stored at the University of Sydney on a secure, password protected backed up database that will be created, much the same as what we have designed for previous University of Sydney studies.

\section{Confidentiality $\{27\}$}

At the hospital, copies of questionnaires will be stored in a locked cabinet. Digital information will be stored on the secure local network. Data will be anonymised before leaving the hospital and being transferred to the University of Sydney for storage on a secure, password-protected drive accessible by authorised researchers only. The anonymisation table will remain at Royal North Shore Hospital.

Plans for collection, laboratory evaluation and storage of biological specimens for genetic or molecular analysis in this trial/future use $\{33\}$ 
Not applicable.

\section{Statistical methods}

\section{Statistical methods for primary and secondary outcomes $\{20 \mathrm{a}\}$}

A continuous series of images from the megavoltage (MV) treatment beam itself will be captured from the electronic portal imaging device (EPID) on the linac. Following the method of Jensen et al. [15], the chest wall position during treatment will be extracted from these images in postprocessing with no additional dose to the patient and no prolongation of treatment time. The planned chest wall position will be extracted from the digitally reconstructed radiograph (DRR). The chest wall displacement for each EPID frame taken during treatment is then defined as the difference of the extracted chest wall position from the planned position.

Deriving an average chest wall displacement per patient we can calculate the systematic error in treatment accuracy, $\Sigma$, as the standard deviation of these values across all patients, as described by Bland and Altman [16]. For each patient the collected respiratory data from both Breathe Well and RPM/RGSC will be recorded, analysed and compared to the ground truth chest wall motion extracted from the cine MV images.

For the secondary aims for the trial, the following data analyses will be carried out. Setup times will be gathered using timepoints recorded in the Aria patient management system and compared for the two trial arms. Questionnaires for patients and staff will be collated and average scores compared for the two systems. Assessment of the dose delivered to patients in each arm will be carried out following the approach of Doebrich et al. [17].

\section{Interim analyses $\{21 \mathrm{~b}\}$}

Not applicable.

\section{Methods for additional analyses (e.g. subgroup analyses) $\{20 \mathrm{~b}\}$}

Not applicable.

Methods in analysis to handle protocol non-adherence and any statistical methods to handle missing data $\{20 \mathrm{c}\}$

Sample size estimation includes an estimation of patients who will be unable to perform DIBH.

\section{Plans to give access to the full protocol, participant level-data and statistical code $\{31 \mathrm{c}\}$}

Only non-identifiable data may be available for other scientific research, e.g., non-identifiable data placed on a well-controlled university site, upon request. The data sharing platform is a secure on-line storage solution ("CloudStor") provided through University of Sydney. The data will be stored as a password- 
protected, encrypted Zip-file. In order to download / decompress the data, participating researchers agree to the terms of use for the data, including: (I) that the data is not to be published or otherwise redistributed without the express consent of the original investigator(s) and (ii) that the data is forbidden to be used for any commercial purpose.

\section{Oversight and monitoring}

\section{Composition of the coordinating centre and trial steering committee $\{5 \mathrm{~d}\}$}

Our steering committee (investigators and sub investigators including consumer representatives) will meet monthly to monitor the conduct of the study and assess progress. In addition, the chief and majority of sub investigators will maintain weekly contact via email and face-face or teleconference meetings in order to facilitate implementation of the study and provide quality assurance to all aspects of the study. The chief investigator will be on-site to personally conduct, oversee, and supervise all of the activities

\section{Composition of the data monitoring committee, its role and reporting structure $\{21 \mathrm{a}\}$}

The imaging modalities that are used in this study are approved for clinical practice, therefore this study we will not nominate a separate Data and Safety Monitoring Board.

\section{Adverse event reporting and harms $\{22\}$}

The principal investigator and sub investigators will report adverse events to the Radiation Safety Officer on site and to the Human Research Ethics Committee and the Research Governance Officer within 72 hours of the event occurring unless immediate notification is required.

\section{Frequency and plans for auditing trial conduct $\{23\}$}

Auditing will only be conducted if required by the funder or Human Research Ethics Committee.

Plans for communicating important protocol amendments to relevant parties (e.g. trial participants, ethical committees) $\{25\}$

Any amendments to the protocol will be reviewed and approved by the Human Research Ethics Committee and communicated to all relevant parties by the lead investigator.

\section{Dissemination plans $\{31$ a $\}$}

The results of this study will be published in internationally recognised peer-reviewed journals and presented at international/national conferences. Non-identifiable data may be available for other scientific research on application to the lead investigator.

\section{Discussion}


Successful completion of this trial and positive results of the primary hypothesis will demonstrate that DIBH with AV biofeedback can be achieved using a simple device without the need for an in-room installation. The ease of use of the Breathe Well device should encourage clinical adoption of this technique to maximise the number of patients who can benefit from DIBH. From a patient perspective, access to a system offering biofeedback allows patients to be active participants in their treatments.

In addition to performing DIBH for left-sided breast cancer patients, a possible future pathway is to investigate the potential of Breathe Well for patients treated in the prone position and for right-sided breast cancer patients, where a reduction in lung dose can be achieved [4].

\section{Trial Status}

Protocol version number: 3.0 (13 August 2020)

Date recruitment began: 24th October 2018

Approximate date when recruitment will be completed: 30th September 2021

The original trial protocol was amended in August 2020 following an interim analysis of the initial 10 patients, with recruitment recommenced in Jan 2021.

\section{Abbreviations}

AV: audio-visual

BRAVEHeart: Breast Radiotherapy Audio Visual Enhancement for sparing the Heart

CT: Computed Tomography

DIBH: deep inspiration breath hold

MV: megavoltage

RPM: real-time position management system (Varian Medical Systems, Palo Alto, CA).

\section{Declarations}

\section{Acknowledgements}

Chris Brown for help with the statistical analysis planning.

\section{Authors' contributions $\{31 \mathrm{~b}\}$}

$H B$ and ES drafted the manuscript and $H B$ will analyse the clinical trial data. $E S, J B, G M, M M, K R, L A, R B$, JA, SS, NP and PK participated in the initial design of this clinical trial with HB and CS contributing to 
amendments. KM, ES and HB developed the software for use in the intervention. CS will oversee the clinical implementation of the Breathe Well technology and will assist HB in collecting the trial data. BZ, FB and HB developed the analysis tools for the trial data. HB and PK developed the statistical methodology for the clinical trial. All authors read and approved the final manuscript.

\section{Funding $\{4\}$}

The BRAVEHeart trial is funded by the National Health and Medical Research Council (NHMRC) Development Grant (APP1073772) and the National Breast Cancer Foundation of Australia Pilot Study Grant (PS-17-055). The funding bodies have no role in the design of the study and collection, analysis, and interpretation of data or in writing the manuscript.

\section{Availability of data and materials $\{29\}$}

Non-identifiable data from the trial will be available from the corresponding author on reasonable request.

\section{Ethics approval and consent to participate $\{24\}$}

The protocol for this clinical trial has been reviewed and approved on 19 June 2015 by the Northern Sydney Local Health District Human Research Ethics Committee (HREC). This HREC is constituted and operates in accordance with the National Health and Medical Research Council's 'National Statement on Ethical Conduct in Human Research' and the CPMP/ICH Note for Guidance on Good Clinical Practice. All patients must provide written informed consent before being enrolled in the trial.

\section{Consent for publication $\{32\}$}

Not applicable.

\section{Competing interests $\{28\}$}

ES, KM and PK are inventors on a patent filed for the Breathe Well technology. KM and PK are founders, shareholders and directors of Opus Medical Pty Ltd, a company advancing the Breathe Well audiovisual biofeedback device.

\section{Authors' information (optional)}

Not applicable.

\section{References}

1. Barton MB, Jacob S, Shafiq J, Wong K, Thompson SR, Hanna TP, et al. Estimating the demand for radiotherapy from the evidence: A review of changes from 2003 to 2012. Radiother Oncol. 2014;112:140-4. 
2. Darby SC, Ewertz M, McGale P, Bennet AM, Blom-Goldman U, Brønnum D, et al. Risk of Ischemic Heart Disease in Women after Radiotherapy for Breast Cancer. N Engl J Med. 2013;368:987-98.

3. Sixel KE, Aznar MC, Ung YC. Deep Inspiration Breath Hold To Reduce Irradiated Heart Volume in Breast Cancer Patients. International Journal of Radiation Oncology^Biology^Physics. 2001;49:199204.

4. Pedersen AN, Korreman S, Nyström H, Specht L. Breathing adapted radiotherapy of breast cancer: Reduction of cardiac and pulmonary doses using voluntary inspiration breath-hold. Radiother Oncol. 2004;72:53-60.

5. Korreman SS, Pedersen AN, Aarup LR, Nøttrup TJ, Specht L, Nyström H. Reduction of cardiac and pulmonary complication probabilities after breathing adapted radiotherapy for breast cancer. International Journal of Radiation Oncology*Biology*Physics. 2006;65:1375-80.

6. Borst GR, Sonke J-J, den Hollander S, Betgen A, Remeijer P, van Giersbergen A, et al. Clinical Results of Image-Guided Deep Inspiration Breath Hold Breast Irradiation. International Journal of Radiation Oncology*Biology*Physics. 2010;78:1345-51.

7. Nissen HD, Appelt AL. Improved heart, lung and target dose with deep inspiration breath hold in a large clinical series of breast cancer patients. Radiother Oncol. 2013;106:28-32.

8. Zellars R, Bravo PE, Tryggestad E, Hopfer K, Myers L, Tahari A, et al. Clinical Investigation: Breast Cancer SPECT Analysis of Cardiac Perfusion Changes After Whole- Breast / Chest Wall Radiation Therapy With or Without Active Breathing Coordinator : Results of a Randomized Phase 3 Trial. Radiation Oncology Biology. 2014;88:778-85.

9. Simonetto C, Eidemüller M, Gaasch A, Pazos M, Schönecker S, Reitz D, et al. Does deep inspiration breath-hold prolong life? Individual risk estimates of ischaemic heart disease after breast cancer radiotherapy. Radiotherapy and Oncology. 2018.

10. Cerviño LI, Gupta S, Rose M, Yashar C, Jiang SB. Using surface imaging and visual coaching to improve the reproducibility and stability of deep-inspiration breath hold for left-breast-cancer radiotherapy. Physics in medicine biology. 2009;54:6853-65.

11. Damkjær SMS, Aznar MC, Pedersen AN, Vogelius IR, Bangsgaard JP, Josipovic M. Reduced lung dose and improved inspiration level reproducibility in visually guided DIBH compared to audio coached EIG radiotherapy for breast cancer patients. Acta Oncol. 2013;52:1458-63.

12. Hu Y, Byrne M, Archibald-Heeren B, Wang Y. Validating the clinical use of Breathe Well, a novel breathe monitoring device. Phys Eng Sci Med. 2020;43:693-700.

13. Chan A-W, Tetzlaff JM, Gøtzsche PC, Altman DG, Mann H, Berlin JA, et al. SPIRIT 2013 explanation and elaboration: guidance for protocols of clinical trials. BMJ. 2013;346:e7586.

14. Jensen C, Urribarri J, Cail D, Rottmann J, Mishra P, Lingos T, et al. Cine EPID evaluation of two noncommercial techniques for DIBH. Med Phys. 2014;41:021730.

15. Bland JM, Altman DG. Statistical methods for assessing agreement between two methods of clinical measurement. Int J Nurs Stud. 2010;47:931-6. 
16. Doebrich M, Downie J, Lehmann J. Continuous breath-hold assessment during breast radiotherapy using portal imaging. Physics Imaging in Radiation Oncology. 2018;5:64-8.

17. Kanphet J, Suriyapee S, Dumrongkijudom N, Sanghangthum T, Kumkhwao J, Wisetrintong M. The systematic and random errors determination using realtime 3D surface tracking system in breast cancer. 2016.

18. Lutz CM, Poulsen PR, Fledelius W, Offersen BV, Thomsen MS. Setup error and motion during deep inspiration breath-hold breast radiotherapy measured with continuous portal imaging. Acta Oncol. 2016;55:193-200.

19. Harris PA, Taylor R, Thielke R, Payne J, Gonzalez N, Conde JG. Research electronic data capture (REDCap)-A metadata-driven methodology and workflow process for providing translational research informatics support. J Biomed Inform. 2009;42:377-81.

20. Harris PA, Taylor R, Minor BL, Elliott V, Fernandez M, O'Neal L, et al. The REDCap consortium: Building an international community of software platform partners. J Biomed Inform. 2019;95:103208.

\section{Figures}

\section{Figure 1}

The RPM and RGSC systems: A) RPM system used at CT. B) RGSC used during linac treatment. C) Patient screen with breath hold position visualisation.

\section{Figure 2}

The Breathe Well device: A) Similar setup on the CT and treatment couch. B) Patient screen with breath hold position visualisation and instructions. 


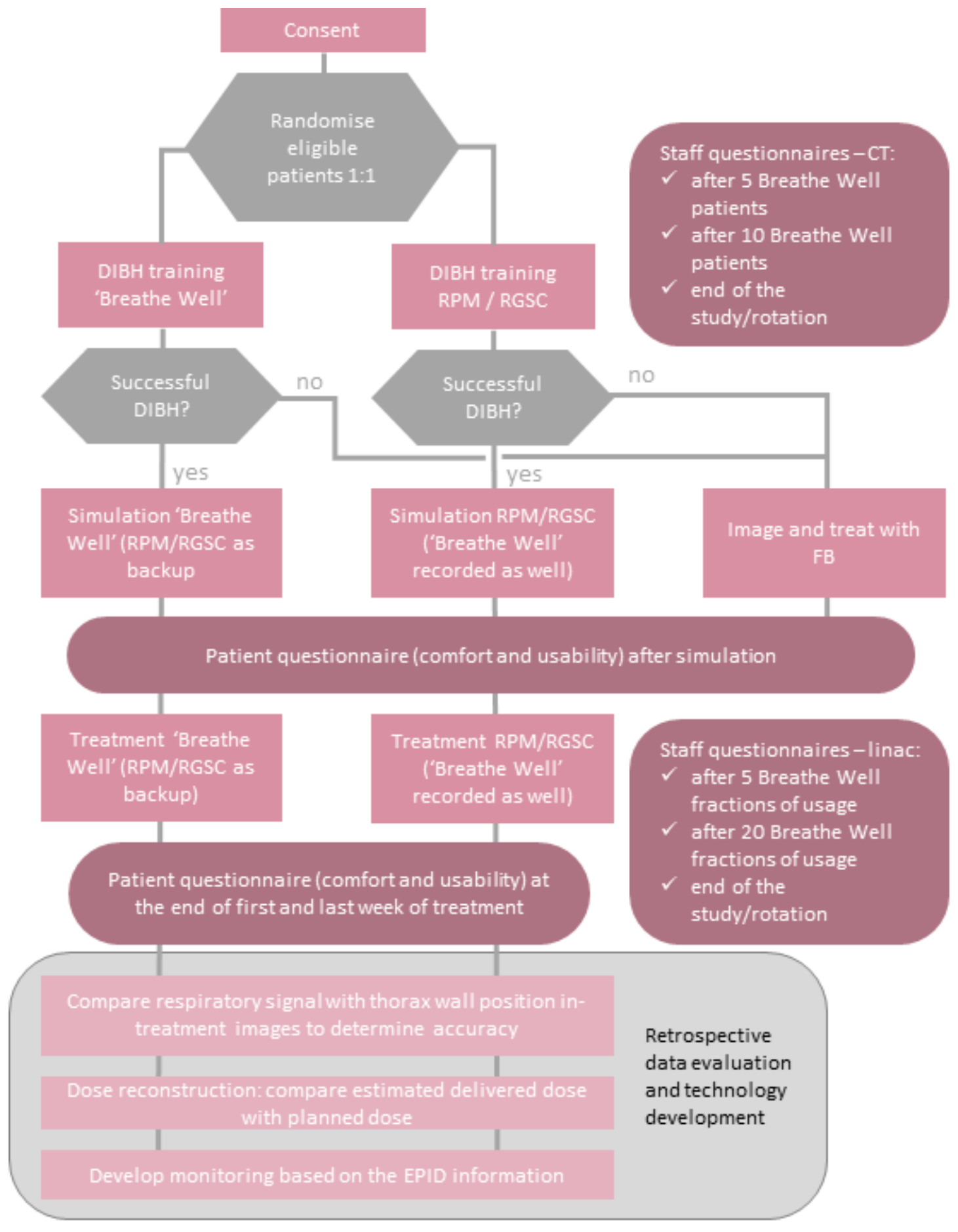

Figure 3

The BRAVEHeart study schema 